\title{
A method for assessing the accuracy of surgical technique in the correction of astigmatism
}

\author{
Stephen B Kaye, Stephen H Campbell, Keith Davey, Allan Patterson
}

\begin{abstract}
Surgical results can be assessed as a function of what was aimed for, what was done, and what was achieved. One of the aims of refractive surgery is to reduce astigmatism; the smaller the postoperative astigmatism the better the result. Determination of what was done - that is, the surgical effect, can be calculated from the preoperative and postoperative astigmatism. A simplified formulation is described which facilitates the calculation (magnitude and direction) of this surgical effect. In addition, an expression for surgical accuracy is described, as a function of what was aimed for and what was achieved.

(Brf Ophthalmol 1992; 76: 738-740)
\end{abstract}

Modern methods of assessing corneal topography have greatly improved the understanding of the changes in corneal curvature which may be induced by surgery. Keratometry, refraction, or computerised corneal topography can be used to measure preoperative $(K 1)$ and postoperative (K3) astigmatism. A mathematical calculation is required merely however to determine the astigmatism induced by the surgery $(K 2)$ - that is, the difference between $K 1$ and $K 3 .{ }^{1}$ It is accepted that it is inadequate to judge surgical effect $(K 2)$ by merely subtracting $K 3$ from $K 1$ without taking the meridions of $K 1$ and $K 3$ into account. ${ }^{1}$

The first part of this communication describes a formula to facilitate the calculation of $K 2$, the measurement of which is necessary to evaluate and audit surgical technique, especially in refractive surgery. The second provides a simple function to assess the accuracy of the surgical technique.

Consider the following example: a patient's preoperative refraction is plano/ +5.00 at $180^{\circ}$ and postoperatively is $+4 /+1.00$ at $180^{\circ}$. The surgical effect $(K 2)$ therefore, was to flatten the horizontal meridian by 4 dioptres - that is, to induce a +4.00 dioptre cylinder at $90^{\circ}$. When $K 1$

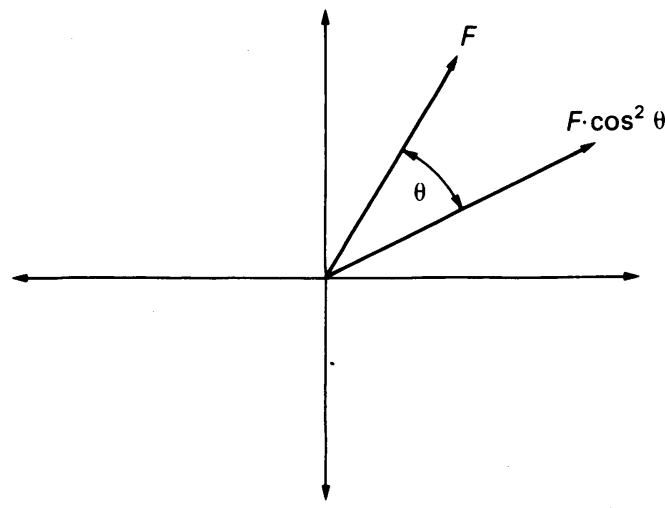

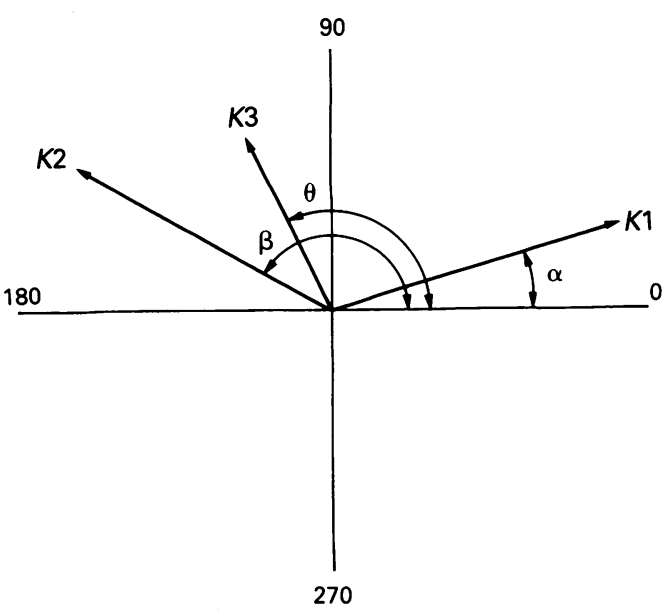

Figure 2 Representation of preoperative astigmatism, $\mathrm{K} 1$ at angle $\alpha$, postoperative astigmatism, $\mathrm{K} 3$ at angle $\theta$, due to the surgical effect, $\mathrm{K} 2$ at angle $\beta$.

and $K 3$ do not lie in the same axis or are not at $90^{\circ}$ to one another, then the calculation of $K 2$ becomes more complex. ${ }^{1}$

In 1975 Jaffe and Clayman' adapted an older method, ${ }^{2-4}$ to calculate $K 2$ in cataract surgery. In essence the power of a cylinder $(F)$ at an angle $(\theta)$ to its principal meridian, is described by the relationship $F \cdot \operatorname{cosine}^{2} \theta$ (Fig 1). Using this relationship they showed that the magnitude and direction $(\beta)$ of $K 2$ can be calculated from $K 1$ and $K 3$ by using the law of cosines (Fig 2 and equations 1 and 2 in Appendix).

This derivation, however, does not readily determine in which quadrant $K 2$ lies - that is, $0^{\circ}$ to $90^{\circ}$ or $90^{\circ}$ to $180^{\circ}$. If $K 1$ is +1.50 dioptres at $25^{\circ}$ and $K 3$ is +3.00 dioptres at $100^{\circ}$ (Fig 3), then from equation $1, K 2$ is plus or minus 4.36

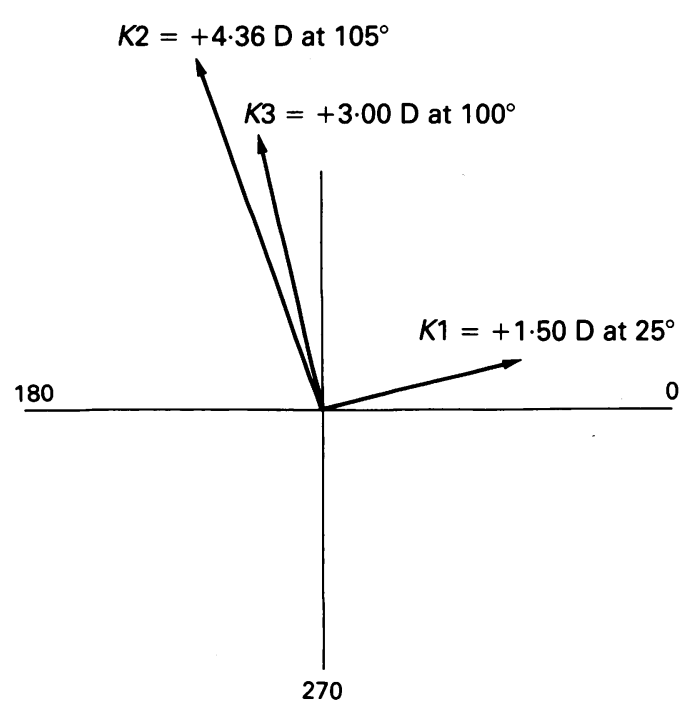

Figure 3 Representation of $\mathrm{K} 2 . \mathrm{K} 1$ is $+1.50 \mathrm{D}$ at $25^{\circ}(\alpha)$, $\mathrm{K} 3$ is $+3.00 \mathrm{D}$ at $100^{\circ}(\theta)$, and $\mathrm{K} 2$ is $+4.36 \mathrm{D}$ and lies in the second quadrant at $104 \cdot 96^{\circ}(\beta)$.
The Unit of

Ophthalmology,

Liverpool L7 8XP

S H Campbel

Mr S B Kaye.

Accepted for publication

17 August 1992 
Figure $4(A) \quad \mathrm{K} 1$ is $+5.00 \mathrm{D} \quad \mathrm{A}$

at $90^{\circ} . \mathrm{K} 2$ is of equal

magnitude to $\mathrm{K} 1$ (that is

$+5 \cdot 00$ D) and placed along the

desired surgical meridian

$\left(90^{\circ}\right.$ to $\left.\mathrm{K} I\right)$ at $0^{\circ}$, so that

$\mathrm{K} 3=0 .(B)$ The effectiveness

of $\mathrm{K} 2$, that is, $\mathrm{K} 2^{\prime} . \mathrm{K} 1$ is

$+5.00 \mathrm{D}$ at $90^{\circ}$ and $\mathrm{K} 2$ is

$+5.00 \mathrm{D}$ at $10^{\circ}$ (that is, $80^{\circ}$ to

$\mathrm{K} 1$, or $10^{\circ}$ away from the

desired meridian) resulting in

a residual astigmatism of

effect of $\mathrm{K} 2$ at $90^{\circ}$ to $\mathrm{K} 1$ -

that is, $\mathrm{K} 2 a^{\prime}-\mathrm{K} 2 b^{\prime}$.
$\mathrm{K} 3=+1 \cdot 74 D . \mathrm{K} 2^{\prime}$ is the net

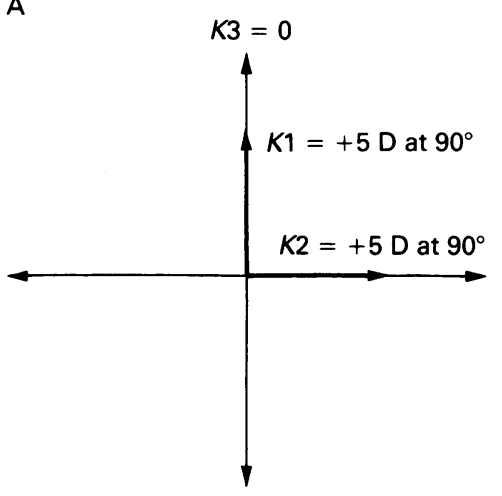

B

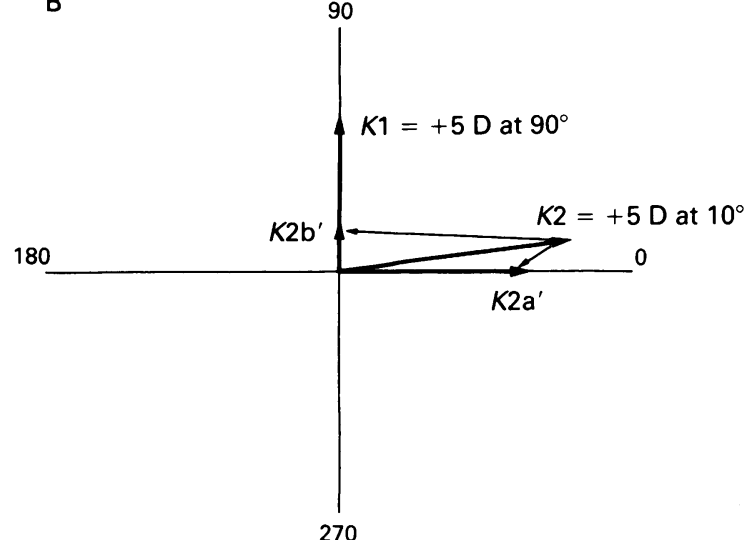

dioptres so that $\beta$ is $14.96^{\circ}$ or $-14 \cdot 96^{\circ}\left(345 \cdot 04^{\circ}\right)$. As shown in Figure 3 however, $K 2$ lies in the second quadrant, therefore $\beta$ is $14.96^{\circ}+90^{\circ}=$ $104 \cdot 96^{\circ}$.

\section{An alternative method for the calculation of $K 2$ and its location}

This communication provides an alternative method enabling the direct calculation of $\beta$ and $K 2$. Consider Figure 2, with $K 1$ at angle $\alpha$ and $K 2$ at angle $\beta$, resulting in $K 3$ at angle $\theta$. It can then be shown that:

$$
\begin{gathered}
K 2=K 3 \cdot \cos 2(\beta-\theta)-K 1 \cdot \cos 2(\beta-\alpha) \\
\text { (Appendix, equation } 3)
\end{gathered}
$$

with $\beta$ calculated directly from equation 4 (see Appendix). If $K 2$ is negative (a minus cylinder), conversion to a positive cylinder can be made by adding $90^{\circ}$ to $\beta$ if $\beta$ is greater than 0 , or subtracting $90^{\circ}$ from $\beta$, if it is less than $0^{\circ}$ (see Appendix).

Given the example above (Fig 3 ), where $K 1$ is $1.5 \mathrm{D}$ at $25^{\circ}$ and $K 3,3 \mathrm{D}$ at $100^{\circ}$, then using equations 3 and $4, K 2$ is $-4.36 \mathrm{D}$ at $14.96^{\circ}$. If preferred, $K 2$ can then be converted to a positive cylinder - that is, $+4.36 \mathrm{D}$ at $104.96^{\circ}$ by adding $90^{\circ}$ to $\beta$.

\section{Assessing the accuracy of refractive surgery}

Methods currently in use to further assess the effects of surgery ${ }^{5}$ are:

1. The vector of flattening $\left(\mathbf{V}_{\mathbf{F}}\right)$ - that is, the steepest preoperative meridian minus the steepest postoperative meridian.

2. The vector of steepening $\left(\mathbf{V}_{\mathbf{S}}\right)$ - that is, the postoperative flat meridian corresponding to the preoperative flat meridian minus the preoperative flat meridian.

3. The flat/steep ratio - that is, $\mathbf{V}_{\mathbf{F}} / \mathbf{V}_{\mathbf{S}}$.

4. The average change in spherical equivalents

Figure 5 Surgical accuracy $(S A)$ as defined by the function $\mathrm{K} 2^{\prime} / \mathrm{K} 2+\mathrm{K} 3$. Graph depicting $S A$ for three values of $\mathrm{K} 2$, as $\mathrm{K} 2$ is rotated away from its optimum meridian. $(A) \mathrm{K} 2=\mathrm{K} 1,(B)$ $\mathrm{K} 2=7 / 5 \mathrm{~K} 1$, and $(C)$ $\mathrm{K} 2=2 / 5 \mathrm{~K} 1$

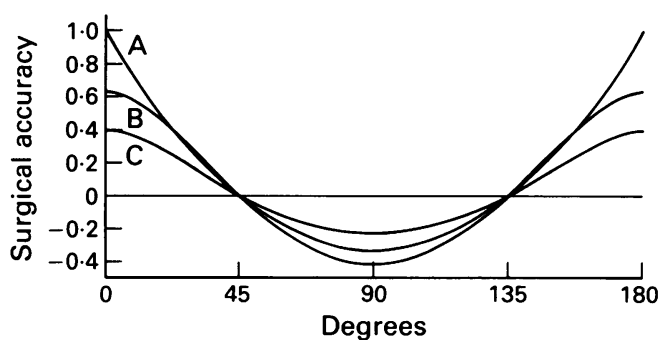

- that is, average postoperative measurements minus average preoperative measurements.

Collectively the size and direction of $K 1$ and $K 3$, the surgical effect, $K 2$, and the overall change in spherical equivalent, give a measure of surgical achievement. It is more difficult however, to provide a single measure which reflects surgical accuracy. $K 3$ by itself provides one measure - that is, the nearer $K 3$ is to zero, the better the surgical achievement. It does not reflect the magnitude of the surgical success (or failure), nor does it indicate whether the surgery was in the desired meridian. Furthermore, the ratio of the pre- and postoperative astigmatism that is, $K 3 / K 1$ does not distinguish, for example, between no effect - that is $K 3=K 1$, or twice the required effect, such that $K 3$ still equals $K 1$. Likewise, the departure from the desired surgical meridian is not indicated.

The ideal surgical meridian of $K 2$ is at $90^{\circ}$ to $K 1$ ( $K 1$ and $K 2$ both deemed positive or negative). Consider the following example, where $K 1$ is $+5 \mathrm{D}$ at $90^{\circ}$ (see Fig 4A). In order to correct this amount of astigmatism, a surgical cylinder of equal magnitude to $K 1$ would need to be placed at $90^{\circ}$ to $K 1$. That is, $K 2=+5 \mathrm{D}$ at $0^{\circ}$ (or $K 2=-5 \mathrm{D}$ at $\left.90^{\circ}\right)$. This would then result in no postoperative astigmatism, that is $K 3=0$ (see Fig 4A).

If $K 2^{\prime}$ represents the net effect of $K 2$ in the desired surgical meridian $\left(90^{\circ}\right.$ to $\left.K 1\right)$ (Appendix equation 5 and Fig $4 \mathrm{~B}$ ), then the ratio $K 2^{\prime} /$ $K 2+K 3$ provides a reasonable surgical accuracy (SA). In the above example, $K 2^{\prime}$ equals $K 2$ (Appendix, equation 5) so that surgical accuracy is:

$$
\mathrm{SA}=+5 \cdot 0 /(+5 \cdot 0+0 \cdot 0)=+1
$$

If, however, surgery resulted in $K 2$ of $+5 \mathrm{D}$ being placed at $10^{\circ}$ (is $80^{\circ}$ to $K 1$, Fig $4 \mathrm{~B}$ ), then the effectiveness of $K 2$ at $0^{\circ}-$ that is $K 2^{\prime}$, in reducing $K 1$ is $+4.67 \mathrm{D}$ in the desired meridian, or $0.33 \mathrm{D}$ less than the ideal (Appendix, equation 5). The resulting postoperative astigmatism is $+1.74 \mathrm{D}$ and SA is $4.67 /(5+1 \cdot 74)=0.69$ (Figs $4 \mathrm{~B}$ and 5).

If $K 2$ is placed nearer the meridian of $K 1$ than the desired meridian, then $K 2^{\prime}$ and therefore $S A$, are less than 0 . This ratio for $\mathrm{SA}$ lies between -0.5 and +1 and reflects the magnitude of overor undercorrection. A negative value also indicates a departure of more than $45^{\circ}$ from the optimum meridian for $K 2$. A result of +1 indicates that both the magnitude and the 
meridian of $K 2$ were optimal. A single measure is thus provided with which to determine surgical accuracy. Figure 5 is a graph showing the effect on $\mathrm{SA}$ as the meridian of $K 2$ is rotated through $180^{\circ}$ for three different values of $K 2$.

The equations for $K 2, b, b$ transposed, $K 2^{\prime}$, and surgical accuracy have been written for use on a Lotus compatible spreadsheet and are given in the Appendix.

1 Jaffe NS, Clayman HM. The pathophysiology of corneal astigmatism after cataract extraction. Trans Am Acad Ophthalmol Otolaryngol 1975; 79: 615-30.

2 Southall JPC. Mirrors, prisms and lenses. A text-book of geometrical optics, 3rd ed. New York: Macmillan, 1940: 320-6.

3 Stokes GG. 19th meeting of the British Association for the Advancement of Science, 1949. Transactions of the Section p 10 (publ 1850).

4 Thompson SP. Obliquely crossed cylindrical lenses. Philosophical magazine (series 5), 1900; xlix: 316-24.

5 Merck PM, Williams PA, Lindstrom RL. Trapezoidal kera totomy. A vector analysis. Ophthalmology 1986; 93: 719-26.

\section{Appendix}

CALCULATION OF $K 2$ AND $b$

$$
K 2^{2}=K 1^{2}+K 3^{2}-2 . K 1 \cdot K 3 \cdot \cos 2(\theta-\alpha)
$$

$K 1$ is the preoperative astigmatism at angle $\alpha$, $K 3$ is the postoperative astigmatism at angle $\theta$, $K 2$ is the surgically induced astigmatism or surgical effect at angle $\beta$, where $\sin 2 \beta=(K 3 \cdot \sin 2 \theta-K 1 \cdot \sin 2 \alpha) / K 2$

From Figure 2, $K 2=\left(K 3 \cos ^{2}(\beta-\theta)-K 1 \cos ^{2}(\beta-\alpha)-K 3 \sin ^{2}\right.$

$$
\left.(\beta-\theta)+K 1 \sin ^{2}(\beta-\alpha)\right)
$$

where

$$
=K 3 \cos 2(\beta-\theta)-K 1 \cos 2(\beta-\alpha)
$$

$$
\begin{aligned}
2 \beta= & \arctan \{(K 1 \sin 2 \alpha-K 3 \sin 2 \theta) / \\
& (K 1 \cos 2 \alpha-K 3 \cos 2 \theta)\}
\end{aligned}
$$

If $K 2$ is negative, conversion to a positive cylinder can be made as stated in the text, by adding or subtracting $90^{\circ}$ to or from $\beta$, if $\beta$ is greater than or less than $0^{\circ}$ respectively. This can be verified by taking the second differential of $K 2$ with respect to $\beta$ - that is,

$$
\begin{aligned}
d^{2} K 2 / d \beta^{2} & =-4 K 3 \cos 2(\beta-\theta)+4 K 1 \cos 2(\beta-\theta) \\
& =-4 K 2
\end{aligned}
$$

Therefore, if $K 2$ is negative, $d^{2} K 2 / d \beta^{2}$ is $>0$ and $K 2$ is a minimum for that value of $\beta$. Adding or subtracting $90^{\circ}$ from $\beta$, will make $K 2$ positive and a maximum, as $d^{2} K 2 / d b^{2}<0$.

SURGICAL ACCURACY

From Figure 4B, the effectiveness of $K 2$ - that is, $K 2^{\prime}$, is $K 2^{\prime}=K 2 \mathrm{a}^{\prime}-K 2 \mathrm{~b}^{\prime}$

$$
\begin{aligned}
K 2^{\prime} & =K 2 \cos ^{2}(\beta-(\alpha+90))-K 2 \cos ^{2}(\beta-\alpha) \\
& =K 2\left[\sin ^{2}(\beta-\alpha)-\cos ^{2}(\beta-\alpha)\right] \\
& =-K 2 \cos 2(\beta-\alpha)
\end{aligned}
$$

Surgical accuracy (SA), can be defined as

$$
\mathrm{SA}=K 2^{\prime} /(K 2+K 3)
$$

FOR USE IN A SPREADSHEET

The following equations, when entered into the appropriate columns of a Lotus compatible spreadsheet, can be used to calculate surgical effect $(K 2)$, its angle $\beta$ and the surgical accuracy.

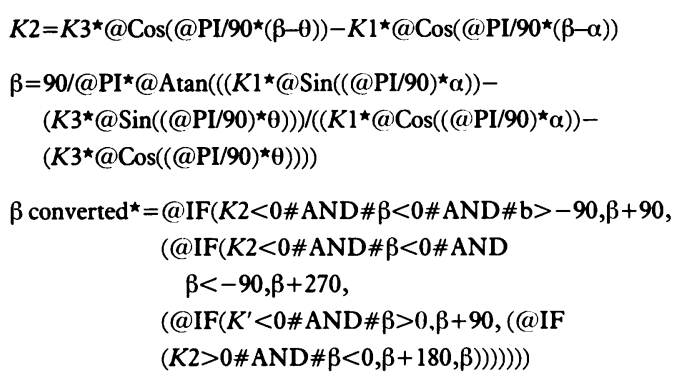

(4)

*this allows $K 2$ to be used as a positive cylinder and expresses $b$ as an angle between $0^{\circ}$ and $180^{\circ}$.

$K 2^{\prime}=K 2^{\star}\left(\left(@ \operatorname{Sin}\left((\mathrm{PI} / 90)^{\star}(\beta-\alpha)\right)\right)^{\wedge} 2-\right.$ $\left.\left(@ \operatorname{Cos}\left((\mathrm{PI} / 90)^{\star}(\beta-\alpha)\right)\right)^{\wedge} 2\right)$

Surgical accuracy $=K 2^{\prime} /(K 2+K 3)$ 